\title{
A GPS Receiver Upgrade For The Space Shuttle - Rationale And Considerations
}

\author{
John L. Goodman* \\ United Space Alliance LLC, Houston, TX 77058
}

\begin{abstract}
In the mid 1990s, a 5 channel Global Positioning System (GPS) receiver was integrated into the Space Shuttle avionics system due to the anticipated start of Tactical Air Control and Navigation (TACAN) phase-out in the year 2000. While the early 1990s technology level receiver adds redundancy and flexibility to the navigation process, and improves safety at emergency landing sites, new capabilities in modern GPS receivers would further enhance Shuttle navigation. All-in-view satellite tracking, new GPS signals and ground and space based augmentation systems would provide a more robust GPS navigation solution for the orbiters, particularly if future missions call for automated landings, or on-board precision orbit determination.
\end{abstract}

\section{Introduction}

$\mathrm{G}$ IPS revolutionized the field of navigation in the 1990s, and made precise navigation available to a wide array of Jusers. Integration of GPS technology into the Shuttle avionics system also occurred in the 1990s. The planned use of GPS during Shuttle missions is not as extensive as some might expect, given the widespread use and success of GPS technology, and the availability of $\sim \$ 100$ receivers to the general public. The rationale behind the current planned use of GPS by the Shuttle Program is discussed along with some history and lessons learned. For the Shuttle Program to fully obtain the benefits of this technology, and to meet any future automation and autonomy requirements, a new GPS receiver with more advanced capabilities than the legacy unit may be needed. ${ }^{1}$

\section{Why GPS?}

As far back as the mid 1970s, the Shuttle Program seriously considered using GPS technology on the orbiters. ${ }^{2}$ GPS technology had the potential to reduce the number of different types of navigation aids on the orbiter, and to make the orbiter more autonomous by providing self contained navigation on-orbit (reduce or eliminate the need for ground radar and Tracking and Data Relay Satellite (TDRS) tracking). However, the developmental nature of GPS technology, adequate performance of the existing Shuttle on-board and ground navigation systems and cost of GPS integration and certification compared to the operational costs of the proven legacy systems drove the Shuttle Program to not incorporate GPS in the 1980s.

By the early 1990s, with the GPS satellite constellation, ground support segment and GPS receivers about to reach the operational phase, government planners began to schedule the phase-out of legacy navigation aids. The Shuttle uses one of these navigation aids, TACAN, during entry. ${ }^{3}$ In 1992, the phase-out of TACAN was anticipated to begin in the year 2000, as indicated in the Federal Radionavigation Plan (FRP). The Shuttle Program initiated an effort that would lead to the replacement of the three TACAN units on each orbiter with three GPS units (the "three string" GPS configuration). ${ }^{4}$

Table 1: Planned TACAN Phase-Out From The Federal Radionavigation Plan

\begin{tabular}{lll}
\hline & & \multicolumn{2}{c}{ Planned Start of } \\
FRP & Publication Date & TACAN Phase-Out \\
\hline 1990 & 1990 & 2000 \\
1992 & June 1993 & 2000 \\
1994 & May 1995 & 2000 \\
1996 & July 1997 & 2005 \\
1999 & December 1999 & 2008 \\
2001 & March 2002 & 2010 \\
$2003^{a}$ & $?$ & $?$ \\
\hline
\end{tabular}

a The 2003 Federal Radionavigation Plan was not available at the time of publication.

Copyright $\odot 2004$ by United Space Alliance, LLC. Published by the American Institute of Aeronautics and Astronautics with permission. These materials are sponsored by the National Aeronautics and Space Administration under Contract NAS9-20000. The U.S. Government retains a paidup, nonexclusive, irrevocable worldwide license in such materials to reproduce, prepare derivative works, distribute copies to the public, and perform publicly and display publicly, by or on behalf of the U.S. Government. All other rights are reserved by the copyright owner.

* Technical Staff, Flight Design and Dynamics, USH-485L, 600 Gemini Ave. Member AIAA. 


\section{Original Shuttle GPS Receiver Selection}

A trade study selected a 5-channel aviation GPS receiver, the Miniaturized Airborne GPS Receiver (MAGR), which entered production in 1994 (Fig. 1). ${ }^{5}$ All-in-view aviation receivers of this type did not become available until the late 1990s. The receiver could be modified to obtain measurements and download data at orbital velocities through software changes, rather than through changes to discrete electronic components, as was required with earlier receivers. The MAGR had several desirable capabilities, including authorized operation and the ability to accept inertial aiding data from an Inertial Navigation System (INS). A change to the receiver power supply and input/output electronics card was required to make the receiver compatible with the orbiter power system and data bus. Radiation testing of the receiver indicated that no hardware changes were required to meet radiation exposure

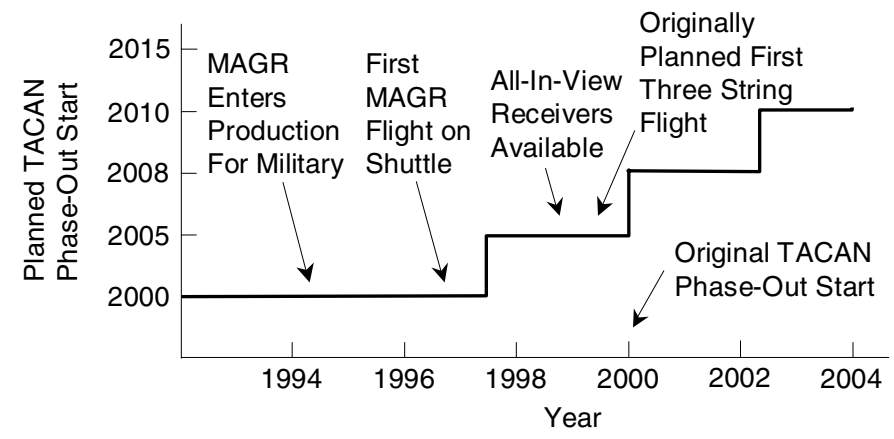

Figure 1: Receiver Selection Driven By Year 2000 Need Date requirements.*

An additional benefit of the MAGR was that it was about to enter mass production. The Shuttle Program could use the existing MAGR logistics base, and take advantage of MAGR software matured by the user community.

During the early to mid 1990s, use of Commercial/Modified Off The Shelf (COTS/MOTS) hardware and software, and the "faster-better-cheaper" approach, were being stressed within NASA. ${ }^{6}$ Use of an off the shelf receiver, and the success of GPS technology, was seen by some as the key to a quick, low cost Shuttle upgrade.

\section{Integration Architecture and Intended Use}

There were a number of ways GPS could have been integrated into the existing on-board navigation system. ${ }^{3,4} \mathrm{~A}$ "parallel navigation" concept was chosen, which avoided changes to the proven entry navigation Kalman filters in the Primary Avionics Software System (PASS) and Backup Flight Software (BFS). This architecture also enabled the same versions of PASS and BFS software to support three different hardware configurations: 1) three TACANs and no GPS units; 2) three TACANs and one GPS unit; or 3) no TACANs and three GPS units. ${ }^{7}$ It also enabled GPS and PASS/BFS data collection to occur while still using the legacy navigation aids for entry. ${ }^{\dagger}$ This was the same architecture that was defined in the late 1970s.

The program requirement for GPS was TACAN replacement. Although the receiver was modified to improve velocity accuracy on-orbit, the improvement was insufficient to facilitate precision orbit determination to the level of accuracy performed by Mission Control (using ground radar and TDRS tracking) to support maneuver planning, debris avoidance analysis and rendezvous. GPS data is used on-orbit during non-rendezvous phases to provide state vector updates to the flight computers.

Use of relative GPS for rendezvous and Ku radar replacement was also a possibility, but was not pursued. Relative GPS requires a GPS equipped target and a spacecraft-to-spacecraft communications link. The program had a requirement to rendezvous with targets that were navigationally passive, such as the Hubble Space Telescope. Furthermore, the International Space Station (ISS) requirements for GPS were limited to coarse orbit determination to support pointing of antennas to the TDRS satellites. Obscuration of GPS signals by ISS structure and multi-path would pose a significant problem for relative GPS in close proximity to the station.

Use of a MAGR modified to perform differential GPS, and replace the Microwave Landing System (MLS), was also discussed, but not pursued due to the continued viability of MLS, and the difficulty of using the five channel

\footnotetext{
* GPS receivers designed for satellites have existed since the early 1990s. However, they do not have features desirable for the Shuttle application which support integration with an INS and atmospheric flight and landing.

$\dagger$ Given the GPS receiver problems that occurred during flight tests, the three year delay in receiver certification, and the Program's decision to fly with three TACANs and one GPS on each orbiter for several years, this choice of architecture was a wise one.
} 
receiver to support the differential application. A differential GPS capability would have required adding Very High Frequency (VHF) antennas to the orbiters, with adequate visibility to support landing, as is done with TACAN antennas. After TACAN replacement, the GPS antenna pairs would occupy two of the positions formerly occupied by TACAN antenna pairs. New antenna positions and associated holes in the orbiter structure for cabling would have been required for the VHF antennas.

\section{The Shuttle and the GPS Experience}

Shuttle test flights of a pre-production MAGR began in December of $1993 .{ }^{8}$ Test flights of a production MAGR modified for the Shuttle application began in September of 1996.* The original flight of three string GPS (no TACANs) was planned for 1999 (Fig. 1). However, performance issues and the slip in TACAN decommissioning caused the Program to schedule several more years of flight and ground testing. ${ }^{9}$ By March of 2002, all four orbiters had flown with one MAGR receiver installed for data collection, and for use in contingencies defined in flight rules. ${ }^{9}$

The MAGR was certified for TACAN replacement in August of 2002. Due to the slip in TACAN phase-out initiation to 2010, the orbiters were anticipated to be flying with three TACAN units and one GPS receiver for some time. Uses for the single GPS receiver configuration, in conjunction with TACAN, on each orbiter were identified, ${ }^{4}$ and the receiver was certified for these applications in December of 2002.

On October 23, 2003, the Shuttle Program approved the removal of the three TACAN units from Endeavour, and the installation of two additional GPS receivers. The first flight of three string GPS is anticipated to occur in 2006.

Other orbiters will be modified as they cycle through the periodic Orbiter Major Modification activity. Until modification, those orbiters flying with three TACAN units and one GPS receiver will incorporate GPS data in parallel with TACAN into the primary and backup flight computers during entry. ${ }^{9}$

\section{Advantages of a New Receiver}

The current receiver represents an early 1990s technology level. Although it has flown on the Shuttle since 1996, and been certified for use during flight, newer GPS units, GPS satellites and augmentation systems are entering (or will soon enter) service with more advanced capabilities. Some of these capabilities could be leveraged to improve Shuttle navigation and safety, particularly if new automation or autonomy requirements emerge (Fig. 2). A study would be required to determine which advanced capabilities would provide benefit to the Shuttle Program. Identification of candidate replacement receivers would be contingent on when new satellite navigation capabilities are introduced, and when receivers that take advantage of those new features are available.

This section discusses several ways in which GPS could be applied to the Shuttle, but not all of the concepts mentioned are desirable for the vehicle.

\section{A. Receiver Recovery}

Modern GPS receivers can track 12 or more GPS satellites at a time. This greatly speeds up state determination after a power cycle or receiver software reset, and reduces the likelihood that difficult tracking conditions will result in unavailability of the GPS navigation solution.

\section{B. Loss of Service}

The five channel unit certified for use on the Shuttle uses four channels to obtain GPS measurements, and a fifth for ephemeris collection, measurement collection for correction of ionospheric errors, tracking channel calibration and satellite acquisition. New satellites needed to maintain track of an optimal set of four are acquired sequentially. Under difficult tracking conditions (multi-path, antenna obscuration, satellite line-of-sight off of the antenna gain pattern), multiple satellite switches could fail or be delayed, resulting in less than optimal satellite geometry or less than four satellite tracking for extended periods of time.

In some cases during testing, the receiver's estimate of its position accuracy exceeded a Shuttle computer GPS quality assurance test limit, which would have prevented the Shuttle computers from using position and velocity data

\footnotetext{
* From STS-79 (September 1996) to STS-107 (January/February 2003), the Shuttle Program has accumulated 7,000 hours of MAGR "nominal performance" operating time from launch through landing. In comparison, 100 hours of TACAN "nominal performance" operating time have been accumulated from Mach 10 through landing from STS-1 (April 1981) through STS-113 (November/December 2002).
} 


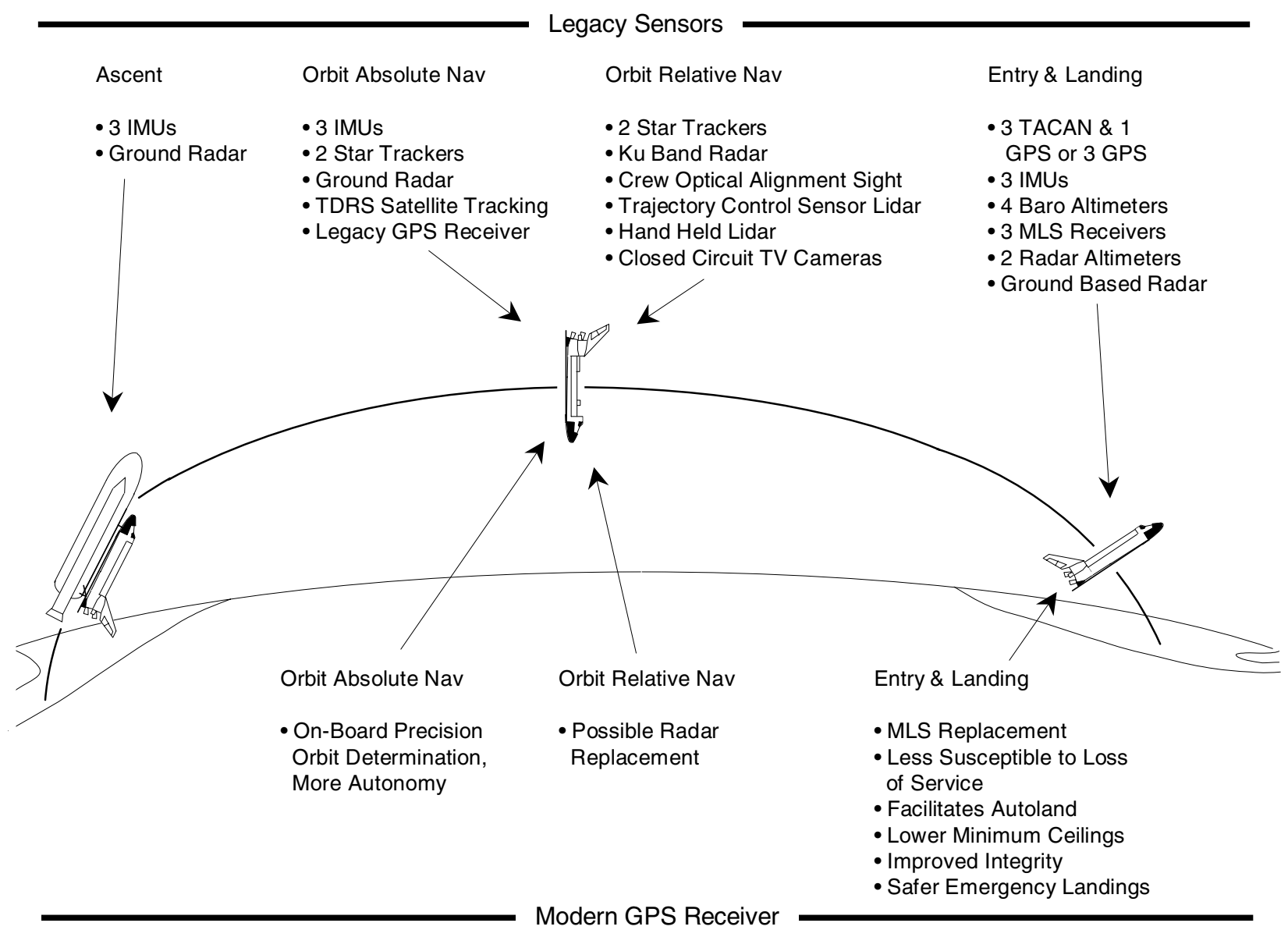

Figure 2: Legacy Navigation Sensors By Flight Phase, With Potential Advantages of a Modern Receiver.

from that receiver. The length of the GPS outages varied. An extensive ground test effort was conducted to ensure that loss of a GPS receiver during entry due to this condition would not compromise safety of flight.

\section{New GPS Signals}

Current GPS satellites broadcast two military signals and one civilian signal, all of which can be tracked by the Shuttle GPS receiver. New GPS satellites, which will be launched in this decade, are the Block IIR-M and Block IIF series. ${ }^{10,11}$

In addition to the three legacy signals, the Block IIR-M satellites will broadcast two new authorized user signals, a new civil signal, and all signals will be broadcast at a higher power level than the previous GPS satellites (Fig. 3). The Block IIF satellites, will broadcast those signals transmitted by the Block IIR-M vehicles, as well as a third civil signal to support safety-of-life applications, such as aircraft. The current Shuttle receiver will be able to track legacy signals transmitted by the Block IIR-M and Block IIF satellites, but a new GPS receiver will be required to take advantage of the additional signals and the improvement they provide to navigation integrity.

Early in the next decade, the next generation GPS satellite, GPS III, may be entering service. This satellite and its new architecture may possess newer signals, enhanced reliability and accuracy.

\section{Receiver Autonomous Integrity Monitoring and Satellite Based Augmentation}

The level of GPS navigation solution accuracy, availability and continuity are important. In addition, the integrity of the navigation solution, whether or not it can be trusted, is critical. ${ }^{12}$ The integrity of the GPS navigation solution is dependant on the on-board GPS receiver, the GPS satellites and the GPS ground support infrastructure.

Currently, the Shuttle Program is dependent on the GPS Master Control Station (MCS) for notification of malfunctioning satellites. If the MCS cannot set the satellite health bit or change the satellite GPS signal to be nontrackable, the MCS would pass word of the malfunctioning satellite to Mission Control. Based on Mission Control 
Block IIR-M Satellites

- M code on L1 \& L2

- Civil signal on L2

- Higher power

- First launch 2004
Block IIF Satellites

- L5 safety of life signal

- Longer lifetime

- First launch 2006
Galileo

- European controlled

- 30 satellites

- Projected full

operational

capability 2008
GPS III

- Fifth generation GPS

- Architecture designed to

support the long term

- Improved accuracy

\& reliability

- New signals?

- First launch 2012?

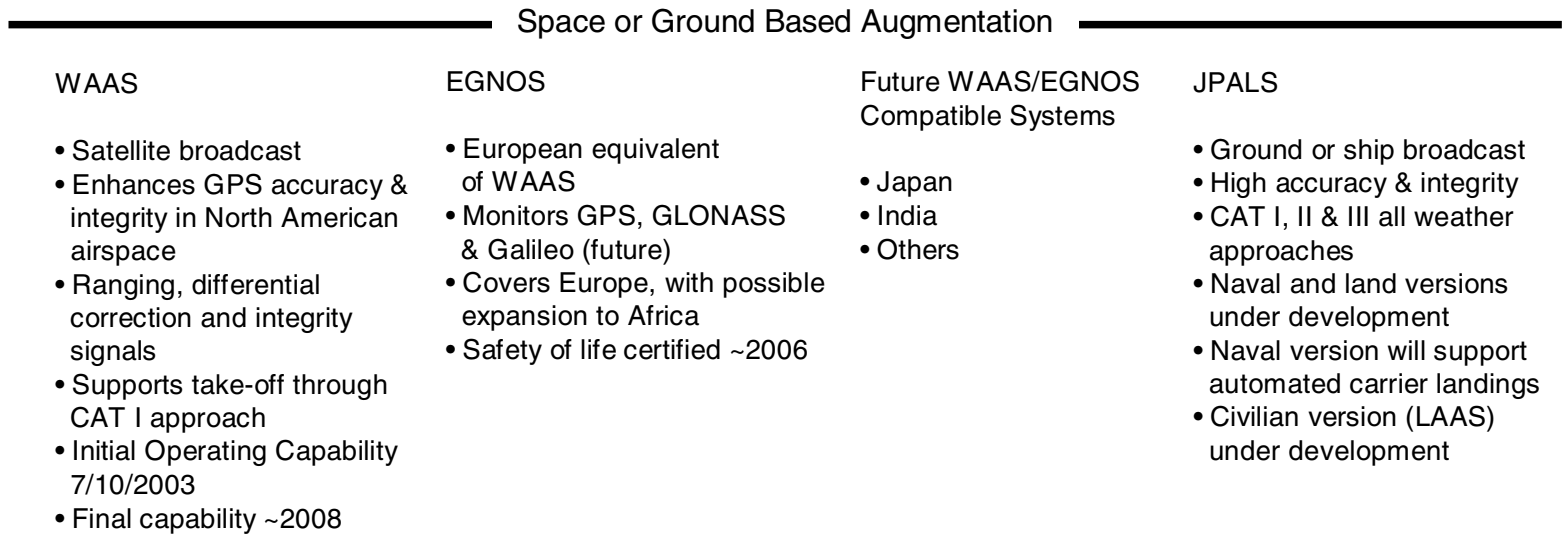

\section{Figure 3: Upcoming Enhancements to Global Navigation Satellite Systems}

instructions, the crew would perform a data entry into a Shuttle computer display to instruct the GPS receivers to ignore the unhealthy satellite.

The all-in-view tracking capability of modern receivers facilitates the use of Receiver Autonomous Integrity Monitoring (RAIM), which detects anomalous GPS signals and identifies suspect GPS satellites, facilitating exclusion of their measurements from state vector determination. The availability of RAIM would provide a level of protection against spurious GPS signals that is not available today with the Shuttle's five-channel receiver.

While RAIM provides protection against suspect satellites, there may be times when a receiver is tracking an insufficient number of GPS satellites with suitable geometry to support RAIM. Satellite or ground based augmentation using ground monitoring stations can provide a higher level of "real time" integrity protection than a stand-alone GPS receiver employing RAIM. ${ }^{12}$

The U.S Wide Area Augmentation System (WAAS) and European Geostationary Navigation Overlay Service (EGNOS), which are satellite based augmentation systems (the augmentation signals are broadcast by satellites other than the GPS satellites), will provide GPS satellite integrity information to those GPS receivers capable of receiving WAAS and EGNOS signals (Fig. 3). ${ }^{13}$ WAAS and EGNOS navigation payloads on geostationary satellites broadcast ranging, differential correction and satellite integrity signals. This will enable receivers to exclude satellites of questionable quality. The additional ranging signals will improve solution quality and availability.

WAAS covers most of North America with GPS corrections and integrity signals, while EGNOS broadcasts GPS and Global Navigation Satellite System (GLONASS) related data to Europe. EGNOS could be expanded to cover Africa, and will eventually broadcast Galileo related data. Other countries such as Japan and India are considering building systems that perform the same function as, and are compatible with, WAAS and EGNOS.

Receiver based RAIM and access to integrity data from satellite based GPS augmentation systems would permit a modern GPS receiver to exclude a questionable satellite in an autonomous manner, more quickly than can be done with the legacy five channel receiver.

\section{E. Shuttle Autoland Using Ground Based GPS Augmentation}

The Shuttle was designed with an autoland system, which has been upgraded over the years. ${ }^{14,15}$ However, a completely automatic landing has never been attempted, and the capability is a back-up flying mode for contingencies.

The Shuttle's MLS is an integral part of the Shuttle's autoland capability. It has been successfully used to support piloted landings since the beginning of the Shuttle flight program in 1981. ${ }^{16}$ The Shuttle MLS system is 
different than that used to support civil aviation, and is only available at six Shuttle landing sites. ${ }^{*}$ The Shuttle has a much steeper glideslope than aircraft. Over the long term, there are difficulties in maintaining the legacy MLS onboard and ground equipment. Multi-path and coverage issues are also of concern with MLS support for autoland.

If automated landings of either a crewed or uncrewed Shuttle are desirable in the future, use of a ground based GPS augmentation system (augmentation signals are broadcast by a ground station) employing the differential GPS concept may be preferable over MLS. A ground-based augmentation system could provide a navigation solution of sufficient accuracy and integrity, provide more operational flexibility in landing site selection, and enhance safety margins.

The military Joint Precision Approach and Landing System (JPALS) ${ }^{\dagger}$ will provide high accuracy, high integrity differential GPS navigation to support precision landing in all weather conditions. ${ }^{17-23}$ Tests of a prototype JPALS system have already supported automated F/A-18 carrier landings and Unmanned Aerial Vehicle (UAV) tests. ${ }^{18,21}$ JPALS will play an important role in future UAV operations. ${ }^{22}$ One set of JPALS ground equipment could service all the runways at a landing site, while the legacy MLS equipment has to be positioned at each runway. This would ensure availability of precision landing capability no matter which landing site runway was selected.

Use of JPALS would require a new GPS receiver. The legacy receiver was not required to, nor does it support, precision landing using GPS augmentation.

\section{F. Weather Minimums For Landings}

Use of an all-in-view receiver with JPALS and RAIM may also permit reduction of minimum ceilings, and provide more safety margin for emergency landings, whether they are piloted or automated.

\section{G. Simplification of Shuttle Navigation Procedures}

A more capable and robust GPS receiver could lead to the simplification of Shuttle on-board and Mission Control navigation procedures. However, any such simplification would have to be proceeded by an extensive analysis of current procedures, Program requirements and flight rules. Furthermore, each proposed change should be examined to determine if the "payback" in terms of life cycle costs and risk reduction justifies the change.

The first measurement processed during entry is drag altitude, computed using Inertial Measurement Unit (IMU) data and an atmosphere model. Drag altitude introduces an expected level of altitude error into the Shuttle navigation state, but bounds altitude errors that are common to inertial navigation systems. All-in-view tracking, RAIM and satellite integrity signals from satellite-based augmentation could eliminate the need for drag altitude measurement processing.

A ground computed correction to the Shuttle on-board navigation state, called a delta state update, is a backup to the on-board navigation system. This capability requires two ground radars, a good Mission Control solution of the Shuttle state vector, and a commanding capability to put the delta-state into the Shuttle computers. Given the integrity functions available to more advanced GPS receivers (WAAS, JPALS, RAIM, more signals), dependence on the delta state backup capability could conceivably be reduced.

Barometric altimeters provide altitude measurements for Kalman filter processing by the Shuttle primary and backup computers. Given that barometric measurements help bound altitude errors at lower altitudes and are more accurate than the previously mentioned drag altitude measurement, it is unlikely that barometric altitude processing would be eliminated. Such a measurement would still be desirable in the event of one or more Shuttle systems problems that result in an unavailability of GPS data. Furthermore, the pitot tubes that provide the barometric altitude measurement also provide data from which several parameters are derived for the orbiter's flight control system.

\section{H. Precision Orbit Determination}

The Shuttle MAGR and ISS Force 19 GPS receivers did not have requirements to support precision orbit determination. While the existing filters in these receivers do meet requirements, flight results show they are not capable of precision orbit determination. ${ }^{24}$ This function is currently performed for the Shuttle by Mission Control, and for the ISS by the U.S. Air Force Strategic Command and the Ballistics Navigation System group at the Russian Mission Control in Korolev. These solutions are used to support maneuver planning and debris avoidance. A Mission Control based filter, called SPOT (Spacecraft Position Optimal Tracking) has been developed to perform

\footnotetext{
* Within the Shuttle Program, the name Microwave Scanning Beam Landing System (MSBLS) is often used to differentiate the Shuttle system from the civil and military aviation Microwave Landing System.

${ }^{\dagger}$ A civilian equivalent, the Local Area Augmentation System (LAAS), is under development.
} 
precision orbit determination using MAGR and Force 19 position vectors. Consideration could be given to providing a precision orbit determination capability on-board the orbiters. This could be done by placing a suitable filtering algorithm in either a GPS receiver, or in a computer external to the receiver. It may be easier to place the orbit determination filter in a computer other than the GPS receiver.

\section{Rendezvous and Proximity Operations}

Application of relative GPS is being pursued by development efforts for the European Automated Transfer Vehicle (ATV) and the Japanese H-II Transfer Vehicle (HTV). Similar application could be considered for the Shuttle as a rendezvous radar replacement.

The Shuttle has a requirement to rendezvous with vehicles that are not equipped with active relative navigation aids such as relative GPS. Any replacement of a current relative sensor with relative GPS would have to be extensively studied to ensure that the Shuttle could still rendezvous with navigationally passive targets.

To support relative GPS for Shuttle rendezvous with the ISS, a new communications link between the ISS and Shuttle may have to be created, with additional hardware and software added to both vehicles. The ISS may also need a new GPS receiver, modified to meet a relative GPS requirement. The current ISS receivers, and the Shuttle's current GPS receiver, were not intended to meet a relative GPS requirement.

Relative GPS could conceivably replace the rendezvous radar and star trackers in their relative sensor roles. Such a replacement would permit similar navigation sensor redundancy (three GPS receivers versus one radar) and eliminate the need for radar failure relative navigation procedures.

The Ku Band antenna and associated electronics would still be required to support transmission of data and video via the TDRS network satellites. In the event of a future communications system upgrade that replaces the Ku Band hardware communications function, replacement of the Ku Band rendezvous function by relative GPS would be desirable. The star trackers used for relative optical measurements are also used for IMU alignment, and would have to be retained in the event of a switch to relative GPS.

Accuracy, multi-path, reliability, antenna obscuration and sensor measurement redundancy concerns in close proximity to the ISS would prevent relative GPS from replacing the laser sensors currently in use to support the close approach and docking phases.

A GPS receiver that is to be used for relative navigation would require extensive modification of navigation and filtering algorithms. If the receiver could not be modified, GPS measurements could be passed to an on-board computer that has navigation and filtering algorithms specifically designed to support relative navigation during rendezvous and proximity operations.

Relative GPS is also more complex than other relative sensors. Given the complexity of the Shuttle and ISS GPS integrations, a relative GPS integration effort should not be considered to be straightforward.

\section{J. Attitude Determination}

Research into GPS attitude determination has been extensive, and such an application has been operational on the ISS since April 2002. ${ }^{25}$ However, for the Shuttle, GPS attitude determination is not desirable.

Multiple antennas are required for GPS attitude determination, and the ISS experience has shown that attitude solution quality is dependent on satellite visibility to the antenna array, which is a function vehicle attitude. Mounting arrays on the Shuttle to ensure adequate visibility would be problematic.

The Shuttle IMUs provide high accuracy attitude data during all phases of flight, at any attitude. The units are aligned using star trackers. The high accuracy is needed to support safety critical ascent and entry, where excessive attitude errors are not permissible. GPS attitude determination may not be of sufficient accuracy to replace the star trackers in the role of supplying attitude updates to the flight computers, for the purpose of IMU alignment.

\section{K. GPS Metric Tracking For Range Safety}

An effort to replace legacy ground radars with on-board GPS receivers for range safety at U.S. launch sites has been underway for some time. Range safety tracking requires two independent sources of tracking data. The Shuttle GPS receiver is integrated with the on-board navigation, guidance and flight control system. This would preclude the use of a new Shuttle GPS receiver from being used to reduce required ground tracking support during launch. An additional GPS receiver, independent of the Shuttle avionics system, would be required to meet range safety requirements for the Shuttle orbiters. Ground radar replacement for range safety would also require the addition of GPS receivers to the Solid Rocket Boosters. In addition to range safety, ground radar tracking is required to support Shuttle launch and landings, which includes nominal and emergency landings at the Kennedy Space Center Shuttle runway. 
Table 2 GPS Flights on the Shuttle and Shuttle Payloads (1993 to 2003) ${ }^{\text {9,24 }}$

\begin{tabular}{|c|c|c|}
\hline Purpose & Unit Flown ${ }^{a}$ & Flights ${ }^{b}$ \\
\hline Space Shuttle & $3 \mathrm{M}$ & $61,59,68,67,69,72,77$ \\
\hline \multirow[t]{2}{*}{$\begin{array}{l}\text { Navigation } \\
\text { Upgrades }\end{array}$} & MAGR & $\begin{array}{l}79,81,82,84,85,89,91,95,88,96,103,99,101,106,92 \\
97,98,102,100,104,105,108,109,110,111,112,113,107\end{array}$ \\
\hline & SIGI (GEM III) & $86,89,91,95,88,96,103$ \\
\hline \multirow[t]{2}{*}{ ISS Testing } & TANS Vector & 77 (GANE) \\
\hline & SIGI (Force 19) & 101 (SOAR), 106 (SOAR) \\
\hline X-38 Testing & SIGI (Force 19) & 100,108 \\
\hline$X-33 \& X-34$ & LN-100G (GEM III) & 81,86 \\
\hline Support & H-764G (GEM III) & 84 \\
\hline Relative GPS & TurboRogue & 69 (WSF-02) \\
\hline Demo & $3 \mathrm{M}$ & 69 \\
\hline ATV Relative & Laben Tensor & 80 (ORFEUS-SPAS 2), 84, $86^{c}$ \\
\hline GPS Demo & TANS Quadrex & 80 \\
\hline Other Payload & Actel & $51 \& 80$ (ORFEUS-SPAS $1 \&$ 2) \\
\hline Support or & Blackjack & 99 (SRTM) \\
\hline Technology & Low Power & \\
\hline Demonstration & Transceiver & 107 (CANDOS) \\
\hline & TANS Quadrex & $51,56,66$ (ATLAS 3) \\
\hline & TANS Vector & 66 \& 85 (CRISTA-SPAS 1 \& 2), 72 (SPARTAN-206 OAS \\
\hline
\end{tabular}

a For Embedded GPS/INS units, the GPS receiver is in parenthesis.

b Payload names are in parenthesis. ATLAS 3, GANE, SOAR, SRTM and CANDOS remained on the orbiter, the others were deployed and retrieved. There may have been other GPS equipped payloads that are not listed.

${ }^{c}$ A Motorola Viceroy receiver was on the Mir space station.

\section{Vulnerability to Space Radiation}

Each succeeding generation of electronic devices, such as GPS receivers, use smaller and smaller electronic components. This may make modern GPS receivers more susceptible to space radiation upsets than the legacy receivers. The higher susceptibility of new receivers to radiation makes use of GPS prior to and during safetycritical flight phases (such as deorbit) questionable. A modern receiver may require expensive modification to meet reliability requirements, or the required reliability may have to be lowered.

\section{Lessons Learned}

Since 1993, numerous flights of GPS receivers have been made on the Space Shuttle (Table 2), in support of Shuttle navigation upgrades, testing for the International Space Station (ISS), X-38, X-33 and X-34 programs, relative GPS technology development, and various other payloads and projects. In addition, GPS has been in operational use on the ISS since April of 2002. GPS units are flying on the Shuttle Training Aircraft, and flew on X38 landing demonstration vehicles when dropped from the NASA B-52B at Edwards Air Force Base. X-38 GPS tests were also conducted on a NASA Beechcraft Beech 200 Super King Air based at the NASA Dryden Research Center. This experience base can be leveraged to mitigate risk inherent in new applications of GPS technology to the Space Shuttle.

The Shuttle, ISS and X-38 GPS projects encountered technical, schedule and budget problems that were not anticipated. ${ }^{9}$ GPS technology proved to be more difficult to apply than was anticipated in the early 1990s. In spite 
of the challenges, the Shuttle and ISS GPS projects eventually resulted in certified GPS receivers for operational use.

A number of lessons were learned during the requirements definition, integration, and testing phases of these projects. ${ }^{26-29}$ Consideration should be given to these lessons learned in any future application of GPS to the Shuttle, or other space vehicles. Independent Verification and Validation ${ }^{30}$ of receiver source code, a close relationship with the vendor and receiver design insight all had to be implemented in the Shuttle GPS receiver project several years before the receiver could be certified for use. Due to lessons learned from the Shuttle GPS integration effort of the late 1990s, a Shuttle Program COTS/MOTS Software Policy document was created. Suggestions contained in this document should be taken under consideration when pursuing a GPS receiver upgrade. ${ }^{31}$

Perhaps the most important lesson concerned people's perception of the maturity of GPS technology for space applications. The widespread success of GPS in myriad applications during the 1990s exceeded the expectations of those in the military navigation arena that designed and developed the system in the 1970s and 1980s. The success seen in terrestrial applications of GPS was anticipated to occur in space applications, but more difficulty in the space applications was encountered than many expected. Optimism about the ease of application of GPS to space flight influenced budget, scheduling and project planning, which led to problems at both the technical and project management levels as the projects progressed.

A paper presented at a 1998 Institute of Navigation conference covered the status and future plans for GPS on spacecraft. Part of the paper discussed reasons for difficulty encountered in development and use of GPS receivers for space flight:

"While this technology holds great promise, its incorporation on spacecraft has been delayed for several reasons. ........ The tremendous success of the very lucrative terrestrial GPS market has, in fact, stifled the development of spaceborne GPS receivers. Companies with GPS expertise are more interested in the lucrative terrestrial-based GPS market. They are not interested in diverting their GPS talent on the relatively small space-based market. This has restricted the development of spaceborne receivers to meet the demands of future spacecraft requirements. " 32

The paper went on to list the reasons why spaceborne GPS receivers have not matured as fast as receivers for terrestrial applications: the perception that GPS technology is mature and requires no research and development to fly in space; more lucrative GPS receiver market for terrestrial-based applications; differences between space-based and ground-based GPS reception; and the requirement for numerous flight experiments to climb the technological stair steps. ${ }^{32}$

As with the legacy aviation receiver currently on the Shuttle, integration of a new receiver, even if in mass production for aviation, should not be regarded as a straightforward task. Caution should be used in trying to leverage aviation certification to achieve certification for the Shuttle.

\section{Conclusion}

The integration of GPS into the Shuttle avionics system added value to the program by providing an eventual replacement for TACAN, a source of state vectors for deorbit in the event of a communications outage, additional redundancy when one receiver is used in conjunction with the legacy TACAN units, more precise navigation when landing at sites with questionable TACAN ground station accuracy, and an additional method of precision orbit determination when GPS position vectors are processed in Mission Control. However, the early 1990s vintage, five channel receiver is vulnerable to "loss of service," cannot support receiver detection and identification of erroneous GPS satellite signals, and cannot support precision landing. A new, more advanced GPS receiver would be required to provide these capabilities, and take advantage of new GPS signals and space and land based augmentation systems. If autonomy and automated landings are a future Shuttle requirement, ${ }^{1}$ a state-of-the-art GPS receiver may be more suitable than the legacy MLS system. Although integration of a new GPS receiver into the Shuttle avionics system would most likely involve a receiver originally designed and mass produced for aviation, the effort should be treated as a development project. Shuttle Program personnel learned many lessons and gained much insight from the Shuttle, ISS and X-38 GPS efforts. ${ }^{26-29}$ This experience could be leveraged to mitigate technical, budget and schedule risk on any future GPS integrations. 


\section{References}

${ }^{1}$ E. Henderson, Bailey, D., and Siders, J., "Space Shuttle Automation Adds Operational Flexibility for Space Transportation Planning," 40th AIAA/ASME/SAE/ASEE Joint Propulsion Conference and Exhibit, AIAA, Reston, VA, 2004.

${ }^{2}$ Van Leeuwen, A., Rosen, E., and Carrier, L., "The Global Positioning System and Its Application In Spacecraft Navigation," Global Positioning System - Papers Published In Navigation, Volume 1, The Institute Of Navigation, Washington, D.C., 1980, pp. $204-221$.

${ }^{3}$ Goodman, J. L., "Space Shuttle Navigation in the GPS Era," Proceedings of the Institute of Navigation National Technical Meeting, Institute of Navigation, Fairfax, VA, 2001.

${ }^{4}$ Goodman, J. L., "Parallel Processing: GPS Augments TACAN in the Space Shuttle," GPS World, Volume 13, Number 10, October 2002, pp. 20-28. See also http://www.gpsworld.com. Accessed on June 16, 2004.

${ }^{5}$ Hoech, R., Bartholomew, R., Moen, V., and Grigg, K., "Design, Capabilities And Performance Of A Miniaturized Airborne GPS Receiver For Space Applications," Proceedings Of The IEEE Position Location And Navigation Symposium, IEEE, New York, NY, 1994.

${ }^{6} \mathrm{McCurdy}, \mathrm{H}$. E., Faster, Better, Cheaper: Low-Cost Innovation in the U.S. Space Program, Johns Hopkins University Press, Baltimore, MA, 2001.

${ }^{7}$ Kachmar, P. M., Chu, W., Neirinckx, P., and Montez, M., "U.S. Space Shuttle - Integrated GPS Navigation Capability," Proceedings of Institute of Navigation GPS Conference, Institute of Navigation, Fairfax, VA, 1993, pp. 313-326.

${ }^{8}$ Madden, C., Lee, S., and Tran, H., "Near Term GPS Application For Shuttle," Proceedings Of The 5th International Technical Meeting Of The Satellite Division Of The Institute Of Navigation, Institute of Navigation, Fairfax, VA, 1992, pp. 863872.

${ }^{9}$ Goodman, J. L., "GPS In Earth Orbit - Experiences From The Space Shuttle, International Space Station And Crew Return Vehicle Programs," Proceedings of the 2002 Core Technologies for Space Systems Conference, Colorado Springs, CO, November 19-21, 2002. See http://www.spacecoretech.org/, Technology Maturation, Transfer, and Utilization Session. Accessed on June 16, 2004.

${ }^{10}$ Shaw, M., Sandhoo, K., and Turner, D., "Modernization of the Global Positioning System," GPS World, Volume 11, Number 9, September 2000, pp. 36-44.

${ }^{11}$ Marquis, W., "M Is For Modernization," GPS World, Volume 12, Number 9, September 2001, pp. 36-42.

${ }^{12}$ Misra, P., and Bednarz, S., "Navigation for Precision Approaches: Robust Integrity Monitoring Using GPS+Galileo," GPS World, Volume 15, Number 4, April 2004, pp. 42-49.

${ }^{13}$ Van Dyke, K., et al, "GPS Integrity Failure Modes and Effects Analysis," Institute of Navigation 2003 National Technical Meeting, January 22-24, 2003, Institute of Navigation, Fairfax, VA.

${ }^{14}$ McWhorter, L. B., and Law, H. G., "Shuttle Autoland Status Summary," AIAA Space Programs and Technologies Conference, AIAA, Reston, VA, 1992.

${ }^{15}$ Tsikalas, G. M., "Space Shuttle Autoland Design," AIAA Guidance and Control Conference, AIAA, Reston, VA, 1982.

${ }^{16}$ Robel, M. C., and Harton, P. L., "Microwave Scanning Beam Landing System Performance During Shuttle Landings," Proceedings of the IEEE National Telesystems Conference, IEEE, New York, NY, 1983.

${ }^{17}$ Stratton, D.A., "Certification and Operational Performance of GPS-Based Landing Systems," Proceedings of the Institute of Navigation 2002 National Technical Meeting, Institute of Navigation, Fairfax, VA, 2002, pp. 507-515.

${ }^{18}$ Sousa, P., Wellons, L., and Colby, G., "Results of Shipboard Relative GPS (SRGPS) Testing for the Pegasus X-47A Unmanned Combat Air Vehicle (UCAV)," Proceedings of the Institute of Navigation 59th Annual Meeting, Institute of Navigation, Fairfax, VA, 2003.

${ }^{19}$ Katanik, T., et al., "Interoperability Between Civil LAAS and Military JPALS Precision Approach and Landing Systems," Proceedings of the Institute of Navigation GPS Conference, Institute of Navigation, Fairfax, VA, 2001, pp. 1179-1189.

${ }^{20}$ Van Dyke, K., Kovach, K., Lavrakas, J., and Carroll, B., "Status Update on GPS Integrity Failure Modes and Effects Analysis," Proceedings of the Institute of Navigation 2004 National Technical Meeting, Institute of Navigation, Fairfax, VA, 2004, pp. 92-102.

${ }^{21}$ Waters, J., Sousa, P., Wellons, L., Colby, G., and Weir, J., "Test Results of an F/A-18 Automatic Carrier Landing Using Shipboard Relative GPS," 57th Annual Meeting of the Institute of Navigation, Institute of Navigation, Fairfax, VA, 2001, pp. 841851.

${ }^{22}$ Croft, J., "Pegasus: UCAVs Look Seaward,” Aerospace America, Vol. 41, No. 9, September 2003, pp. 36-42.

${ }^{23}$ Pervan, B., Chan, F., Gebre-Egziabher, D., Pullen, S., Enge, P., and Colby, G., "Performance Analysis of Carrier-Phase DGPS Navigation for Shipboard Landing of Aircraft," Navigation: Journal of the Institute of Navigation, Volume 50, Number 3, Fall 2003, Institute of Navigation, Fairfax, VA, pp. 181-191.

${ }^{24}$ Carpenter, J. R., and Schiesser, E. R., "Semimajor Axis Knowledge and GPS Orbit Determination," Navigation: Journal of the Institute of Navigation, Volume 48, Number 1, Spring 2001, Institute of Navigation, Fairfax, VA, pp. 57-68.

${ }^{25}$ Gomez, S. F., "Flying High - GPS on the International Space Station and Crew Return Vehicle," GPS World, Volume 13, Number 6, June 2002, pp. 12-20. See also http://www.gpsworld.com. Accessed on June 16, 2004. 
${ }^{26}$ Goodman, J. L., "Lessons Learned From Flights of "Off the Shelf” Aviation Navigation Units on the Space Shuttle," Joint Navigation Conference, Orlando, Florida, May 6-9, 2002. See also Lesson \#1370 on the NASA Public Lessons Learned System Database website: http://lis.nasa.gov/llis/plls/index.html. Accessed on June 16, 2004.

${ }^{27}$ Goodman, J. L., "The Space Shuttle and GPS - A Safety-Critical Navigation Upgrade," COTS-Based Software Systems, edited by H. Erdogmus and T. Weng, Springer-Verlag Lecture Notes in Computer Science Volume 2580, Springer-Verlag, Berlin, Germany, 2003, pp. 92-100.

${ }^{28}$ Goodman, J. L., “A Software Perspective On GNSS Receiver Integration and Operation,” Satellite Navigation Systems: Policy, Commercial and Technical Interaction, edited by M. Rycroft, Kluwer Academic Publishers, Dordrecht, The Netherlands, 2003, pp. 119-126.

${ }^{29}$ Gomez, S., and Lammers, M. L., "Lessons Learned From Two Years of On-Orbit Global Positioning System Experience on International Space Station," Proceedings of the Institute of Navigation Global Navigation Satellite Systems Conference (GNSS 2004), Institute of Navigation, Fairfax, VA, 2004.

${ }^{30}$ Beims, M. A., and Dabney, J. B., "Reliable Tailored-COTS Via Independent Verification and Validation," NATO Symposium: Commercial Off-The-Shelf Products in Defence Applications, Brussels, Belgium, April 3-7, 2000.

31"CCommercial Off-the-Shelf (COTS), Modified Off-the-Shelf (MOTS) Software Policy, SSP Directive No. 145," dated April 16, 2001, in Space Shuttle Program Structure And Responsibilities, Book 2, Space Shuttle Program Directives, NSTS 07700, NASA Johnson Space Center.

${ }^{32}$ Bauer, F. H., Hartman, K., and Lightsey, E. G., "Spaceborne GPS Current Status and Future Visions," Proceedings of Institute of Navigation GPS Conference, Institute of Navigation, Fairfax, VA, 1998, pp. 1493-1508. 